\title{
An Investigation of Molecular Templating in Amorphous Silicas by Cross-Polarization NMR Spectroscopy
}

\author{
C. A. Click, ${ }^{1}$ R. A. Assink, ${ }^{2}{ }^{*}$ C. J. Brinker, ${ }^{2,3}$ and S. J. Naik ${ }^{3}$ \\ 1. University of Missouri, Rolla, MO 65401 \\ 2. Sandia National Laboratories, Albuquerque, NM 87185-1407 \\ 3. University of New Mexico, Albuquerque, NM 87131
}

\begin{abstract}
The precise pore sizes defined by crystalline zeolite lattices have led to intensive research on zeolite membranes. Unfortunately zeolites have proven to be extremely difficult to prepare in a defect-free thin film form needed for membrane flux and selectivity. We introduce tetrapropylammonium (TPA), a structure-directing agent for zeolite ZSM-5, into a silica sol and exploit the development of high solvation stresses to create templated amorphous silicas with pore apertures comparable in size to those of ZSM-5. Silicon and carbon NMR experiments were performed to evaluate the efficacy of our templating approach. The ${ }^{29} \mathrm{Si}$ NMR spectrum of the silica matrix was observed by an intermolecular cross-polarization experiment involving the ${ }^{1} \mathrm{H}$ nuclei of TPA and the ${ }^{29} \mathrm{Si}$ nuclei in the silica matrix. The efficiency of the cross-polarization interaction was used to investigate the degree to which the matrix formed a tight cage surrounding the template molecule. Bulk xerogels, prepared by gelation and slow drying of the corresponding sols, exhibited only weak interactions between the two sets of nuclei. Thin film xerogels, where drying stresses are greater, exhibited significantly increased interactions. Intramolecular crosspolarization experiments between the ${ }^{1} \mathrm{H}$ and ${ }^{13} \mathrm{C}$ nuclei of the template molecule demonstrated that much of the increased efficiency was a result of reduced rotational mobility of the TPA molecule.
\end{abstract}

TECEIVED

JUL 21

1999

OSTI

Sandia is a multiprogram laboratory operated by Sandia Corporation, a Lockheed Martin Company, for the United States Department of Energy under Contract DE-AC0494AL85000. 


\section{DISCLAIMER}

This report was prepared as an account of work sponsored by an agency of the United States Government. Neither the United States Government nor any agency thereof, nor any of their employees, make any warranty, express or implied, or assumes any legal liability or responsibility for the accuracy, completeness, or usefulness of any information, apparatus, product, or process disclosed, or represents that its use would not infringe privately owned rights. Reference herein to any specific commercial product, process, or service by trade name, trademark, manufacturer, or otherwise does not necessarily constitute or imply its endorsement, recommendation, or favoring by the United States Government or any agency thereof. The views and opinions of authors expressed herein do not necessarily state or reflect those of the United States Government or any agency thereof. 


\section{DISCLAIMER}

Portions of this document may be illegible in electronic image products. Images are produced from the best available original document. 


\section{Introduction}

There is intense interest in the use of zeolites in the form of membranes for gas separation or pervaporation applications because of their ordered and uniform pore structure. ${ }^{1-3}$ Zeolites are crystalline materials, however, and are difficult to prepare as defect-free thin films needed for separation applications. We wish to use a molecular templating approach ${ }^{4}$ to form well-defined porosity in amorphous silica matrices prepared by the solgel method. ${ }^{5}$ The tetrapropylammonium (TPA) cation, used as a structure-directing agent in ZSM-5 synthesis, ${ }^{4}$ was incorporated as a template within a sol-gel silica matrix. We exploit the development of high solvation stresses to create templated amorphous silicas with pore apertures comparable in size to those of ZSM-5. Organic-inorganic hybrid materials are formed which on calcination result in TPA-templated microporous silica (figure 1). The corresponding membranes are ultrathin, have low defect density and show molecular sieving behavior. This paper utilizes the efficiencies of intermolecular and intramolecular cross-polarization NMR experiments to characterize the effectiveness of the templating process. Creation of a convenient method to measure templating effectiveness will aid in optimizing the entire templating process.

Previously Lefebvre et. al. ${ }^{6}$ measured the intermolecular cross-polarization between the ${ }^{1} \mathrm{H}$ nuclei in p-xylene and the ${ }^{29} \mathrm{Si}$ nuclei in ZSM-5. They showed that the crosspolarization efficiency for the various silicons depended on their proximity to a ${ }^{1} \mathrm{H}$ nucleus in the occluded p-xylene molecule. The cross-polarization transfers were most efficient when the ${ }^{1} \mathrm{H}$ and ${ }^{29} \mathrm{Si}$ nuclei were separated by distances of approximately $3.3 \mathrm{~A}$ or less. Recently Burkett and Davis used intermolecular ${ }^{1} \mathrm{H}$ to ${ }^{29} \mathrm{Si}$ cross-polarization NMR to study the interactions between tetrapropylammonium (TPA) and the ZSM-5 zeolite matrix. They show that as the gel is heated, the intermolecular cross-polarization efficiency quickly increases, providing evidence that structure direction occurs early in the synthetic process.

The cross-polarization efficiency between two nuclei, for example between ${ }^{1} \mathrm{H}$ and $\mathrm{X}$, where in this study $\mathrm{X}$ is either ${ }^{29} \mathrm{Si}$ or ${ }^{13} \mathrm{C}$, depends on the strength of the internuclear interactions and the time dependence of fluctuations in the internuclear interactions. ${ }^{8}$ The buildup in polarization of the $\mathrm{X}$ nuclei is most efficient when the internuclear distances are short. The cross-polarization rate is proportional to the inverse of the ${ }^{1} \mathrm{H}-\mathrm{X}$ distance raised to the sixth power. Simplified expressions for the time dependence of the fluctuations show that the cross-polarization rate is proportional to the correlation time 
constant, $\tau_{c}$, describing the interaction fluctuations. Thus relatively rigid, slow moving systems with long correlation time constants cross-polarize most efficiently.

Templating effectiveness, which we define as the degree to which the matrix forms a tight cage surrounding the template molecule, is expected to affect both the internuclear distances and the correlation time constant used to describe the intermolecular crosspolarization experiment. As the templating effectiveness is increased, we expect that both factors will change in a direction to increase the cross-polarization efficiency. Thus overall cross-polarization efficiency can be used to measure the relative templating effectiveness. In this study we have prepared two silica matrices in which TPA was used as a templating molecule. For bulk xerogels, slow gelation and drying allow significant time for condensation reactions to occur. This limits the extent of collapse of the gel network in response to the capillary pressure during drying. For thin film xerogels, solvent evaporation and drying occur simultaneously in the short time scale of the drying process. These materials are therefore expected to show greater extent of shrinkage of the network around the organic template molecules. We find that the thin film sample exhibits increased ${ }^{1} \mathrm{H}^{29} \mathrm{Si}$ intermolecular cross-polarization efficiency corresponding to improved templating effectiveness. Additional ${ }^{1} \mathrm{H}-{ }^{13} \mathrm{C}$ intramolecular cross-polarization experiments show that the changes in efficiency are primarily related to changes in the correlation time describing the rotational motion of the TPA molecule.

\section{Experimental Section}

Synthesis. Precursor silica sols were prepared by a two-step acid catalyzed hydrolysis and condensation process. Tetraethylorthosilicate (TEOS, 98\%, Aldrich), ethanol, deuterated water $\left(\mathrm{D}_{2} \mathrm{O}, 99.9\right.$ atom\% D, Cambridge Isotope Laboratories) and $\mathrm{HCl}$ were initially refluxed at $60^{\circ} \mathrm{C}$ for $90 \mathrm{~min}$. In the second step, additional $\mathrm{D}_{2} \mathrm{O}$ and $\mathrm{HCl}$ were added to make up the final mole ratio of $1.0: 3.8: 5.0: 0.004$ (TEOS:EtOH: $\mathrm{D}_{2} \mathrm{O}: \mathrm{HCl}$ ) and the solution was shaken for 15 minutes at room temperature. Tetrapropylammonium bromide (TPABr, 98\%, Aldrich) was added as $4 \%$ by weight of the silica sol.

Bulk xerogel samples were prepared by gelation and slow drying of these composite sols at $50^{\circ} \mathrm{C}$. These were then ground to a fine powder. For preparing xerogels in thin film form, the sols were diluted with ethanol in the ratio 2:1 (vol. EtOH : vol. sol). These diluted sols were then cast as thin films in glass petridishes and dried at room temperature. Both the bulk and thin film xerogels were finally dried at $150^{\circ} \mathrm{C}$ for 3 hours. 
Spectroscopy. NMR spectroscopy was performed on a Bruker AMX-400 equipped with a $7 \mathrm{~mm}$ CP MAS probe. The ${ }^{29} \mathrm{Si}$ and ${ }^{13} \mathrm{C}$ spectra were recorded at 79.5 and $100.6 \mathrm{MHz}$, respectively, using zirconate rotors spinning at $4 \mathrm{kHz}$. The ${ }^{29} \mathrm{Si}$ spectra were calibrated by setting the lowfield resonance of octakis (trimethylsiloxy) silsesquioxane to $12.5 \mathrm{ppm}$ and the ${ }^{13} \mathrm{C}$ spectra were calibrated by setting the lowfield resonance of a glycine sample to $176.0 \mathrm{ppm}$. The ${ }^{29} \mathrm{Si}$ cross-polarization spectra were recorded with 1 and $3 \mathrm{~ms}$ contact times, a 4 s delay time and 512 scans. The ${ }^{13} \mathrm{C}$ direct spectra were recorded with delay times ranging from 5 to $20 \mathrm{~s}$ and 256 scans. The ${ }^{13} \mathrm{C}$ cross-polarization spectra were recorded with 0.3 and $1 \mathrm{~ms}$ contact times, a $4 \mathrm{~s}$ delay time and 256 scans.

\section{Results}

The ${ }^{1} \mathrm{H}-{ }^{29} \mathrm{Si}$ NMR intermolecular cross-polarization spectra of the bulk and thin film xerogels for cross-polarization contact times of 1 and $3 \mathrm{~ms}$ are shown in Figure 2. These contact times span the range of maximum intensity, which occurs between 2 and $3 \mathrm{~ms}$ for each of the samples. The signal intensities of the bulk samples are less than those of thin film samples for both contact times. Since these differences are critical for our analysis, the experiments were repeated with repacked sample rotors and with careful tuning of the probe before each experiment. In order to quantify the cross-polarization efficiency, the resonances for each sample were integrated and normalized by their respective weights. The normalization factors were small since the weight difference between samples was less than $5 \%$ for each set. The normalized ratios of signal intensities for the normally dried and reduced pressure samples are shown in Table I.

We were concerned that the two methods of preparation may result in differing concentrations of TPA molecules. The apparent differences in cross-polarization efficiencies could then be attributed to differences in the number of TPA hydrogens that are available to cross-polarize the silicon nuclei. The ${ }^{13} \mathrm{C}$ spectrum of each sample was recorded by direct polarization in order to determine its relative TPA concentration. Delay times of $5 \mathrm{~s}$ and longer were found to provide spectra of constant intensity. The total intensities of spectra recorded at delay times of 10 and $20 \mathrm{~s}$ were combined and again normalized by the appropriate sample weights. The concentrations of TPA in the two samples are the same within experimental error. The results are summarized in Table II. 
The intramolecular ${ }^{1} \mathrm{H}-{ }^{13} \mathrm{C}$ cross-polarization spectra of TPA were recorded to look at the cross-polarization efficiency within the TPA molecule. Spectra with contact times of 0.3 and $1 \mathrm{~ms}$, which spanned the maximum cross-polarization efficiency, were recorded. Spectral intensities showed that the intramolecular cross-polarization efficiency of the thin film sample is greater than that of the bulk xerogel. The results, normalized for slightly differing sample weights, are shown in Table I.

\section{Discussion}

The efficiency of intermolecular cross-polarization of the silicon nuclei in the silica matrix by the hydrogens in the TPA template molecule was used to probe the degree to which templating was accomplished. More effective templating leads to greater intermolecular cross-polarization efficiency. The relationship between templating and intermolecular cross-polarization depends on two factors: (1) better templating reduces the distance between the hydrogen nuclei on the TPA molecule and the silicon nuclei in the silica matrix and (2) better templating is also expected to restrict the rotational motion of the TPA molecule and thus increase the correlation time constant describing the internuclei fluctuations. Each of these effects contributes to an increase in crosspolarization efficiency with increasing templating effectiveness.

The ${ }^{1} \mathrm{H}^{29} \mathrm{Si}$ intermolecular cross-polarization efficiency of the thin film xerogel is greater than that of the bulk xerogel sample for cross-polarization times less than or equal to the time for maximum polarization transfer. The signal intensities were adjusted by their respective sample weights. Direct polarization ${ }^{13} \mathrm{C}$ spectra indicated that the thin film sample had a slightly lower concentration of TPA molecules. After these adjustments were applied, the cross-polarization efficiency of the thin film sample was 35\% greater than that of the bulk sample. The greater cross-polarization efficiency demonstrates that the templating process is improved in the thin film sample. The improvement can be attributed to the greater drying stresses during thin film formation.

Intramolecular ${ }^{1} \mathrm{H}-{ }^{13} \mathrm{C}$ cross-polarization spectra of the TPA molecule were recorded in order to determine the relative importance of changes in the motion describing the internuclear fluctuations. The ${ }^{13} \mathrm{C}$ spectra show that intramolecular cross-polarization for the thin film sample is more efficient than that for the bulk. Under identical spectral conditions, the thin film sample has $26 \%$ greater polarization compared to the bulk xerogel. This increase in polarization must be attributed entirely to an increase in the correlation time constant describing the change in orientation of the $\mathrm{C}-\mathrm{H}$ vector with 
respect to the external magnetic field. Changes in internuclear distances could not contribute to the increase since the distances for the intramolecular cross-polarization process are identical for the two samples.

The motions of the TPA molecule can be classified as translational, internal rotations of the propyl chains and overall rotational motion of the TPA molecule. Translational motion of TPA does not affect the magnitude or orientation of the ${ }^{1} \mathrm{H}^{13} \mathrm{C}$ intramolecular vectors and therefore cannot affect the efficiency of the cross-polarization process. The fast internal motions of the propyl chains reduce the internuclear interactions by a constant factor, which is a function of the angle between the ${ }^{1} \mathrm{H}-{ }^{13} \mathrm{C}$ vector and the axis of rotation. To the extent to which these internal motions depend primarily on the sample temperature and internal barriers to rotation, they would be similar for the two samples. The difference in effective correlation times then depends on the difference in the overall slow tumbling of the TPA molecule. The ${ }^{13} \mathrm{C}$ cross-polarization experiment shows that the overall rotational motion of TPA is more restricted in the thin film sample.

The rotational correlation times describing the fluctuations of ${ }^{1} \mathrm{H}-{ }^{13} \mathrm{C}$ interactions in the intramolecular experiments should also modulate the ${ }^{1} \mathrm{H}^{29} \mathrm{Si}$ interactions in the intermolecular experiments. The rotational motion of the TPA molecule would move a hydrogen nucleus completely in or out of the sphere of influence of a silicon nucleus fixed in the silica matrix. Thus, we expect that the decrease in rotational mobility of the TPA molecule in the thin film sample accounts not only for all of the increased efficiency of the ${ }^{1} \mathrm{H}-{ }^{13} \mathrm{C}$ cross-polarization experiment, but also for much of the increased efficiency of the ${ }^{1} \mathrm{H}-{ }^{29} \mathrm{Si}$ cross-polarization experiment. The remaining difference between the $35 \%$ efficiency of the intermolecular and $27 \%$ efficiency of the intramolecular experiments would be attributed to changes in translational mobility and decreases in the internuclear distances.

We believe that the close association between improved templating, increased intermolecular cross-polarization efficiency and an increase in the molecular rotation correlation time is reasonable. The TPA molecule has a relatively spherical shape. Small changes in cavity size are expected to have a dramatic effect on its rotational freedom. For example, molecular crystals often undergo phase transitions from static molecules to freely rotating molecules over a narrow temperature range. Fullerene $\mathrm{C}_{60}$ has been shown to undergo a solid-solid phase transition at $260 \mathrm{~K}$ during which the rotational mobility of the molecules increases by over three orders of magnitude. ${ }^{9,10}$ This transition is 
accompanied by a discontinuous increase in lattice spacing from 14.1015 to $14.1501 \mathrm{~A}$, an increase of only $0.0486 \mathrm{~A} .^{11}$

\section{Summary}

We have found the intermolecular ${ }^{1} \mathrm{H}-{ }^{29} \mathrm{Si}$ cross-polarization efficiency increases during sol-gel thin film formation indicating an improved effectiveness of molecular templating. Much of the increased cross-polarization efficiency can be attributed to a reduction in the rotational mobility of the TPA templating molecule. Since the rotational motion and the templating efficiency are so closely coupled, these results suggest that the ${ }^{1} \mathrm{H}-{ }^{13} \mathrm{C}$ intramolecular cross-polarization behavior of the TPA molecular can also be used to monitor templating efficiency. The analysis is then reduced to recording the relative intensities of the ${ }^{1} \mathrm{H}-{ }^{13} \mathrm{C}$ intramolecular cross-polarization spectra and normalizing these intensities by the TPA concentrations determined from the direct ${ }^{13} \mathrm{C}$ spectra. Alternatively, if deuterated TPA is used in the synthesis, the deuterium lineshape provides an accurate probe of the TPA rotational motion. Preliminary lineshape experiments have been encouraging.

\section{References}

1. Bein, T. Chem. Mater. 1996, 8, 1636.

2. Den Exter, M. J.; Jansen, J. C.; Van De Graff, J. M.; Kapteijn, F.; Moulijn, J. A.; Van Bekkum, H. Stud. Surf. Sci. Catal. 1996, 102, 413.

3. Matsukata, M.; Kikuchi, E. Bull. Chem. Soc. Jpn. 1997, 70, 2341.

4. Flanigen, E. M.; Bennett, J. M.; Grose, R. W.; Cohen, J. P.; Patton, R. L.; Kirchner, R. M.; Smith, J. V. Nature (London) 1978, 271, 512.

5. Brinker, C. J.; Scherer G. W. Sol-Gel Science: The Physics and Chemistry of Sol-Gel Processing, Academic Press, Inc.: San Diego, 1990.

6. Lefebvre, F.; Sacerdote-Peronnet, M.; Mentzen, B. F. C. R. Acad. Sci. Paris, Ser. 2 $1993,316,1549$.

7. Burkett, S. L.; Davis, M. E. J. Phys. Chem. 1994, 98, 4647.

8. Mehring, M. In NMR - Basic Principles and Progress, Vol. 11; Diehl, P., Fluck, E., Kosfeld, R., Eds.; Springer-Verlag: Berlin, 1976; p.112-153.

9. Yannoni, C. S.; Johnson, R. D.; Meijer, G.; Bethune, D. S.; Salem, J. R. J. Phys. Chem. 1991, 95, 9.

10. Tycko, R.; Haddon, R. C.; Dabbagh, G.; Glarum, S. H.; Douglass, D. C.; Mujsce, A. M. J. Phys. Chem. 1991, 95, 518.

11. David, W. I. F.; Ibberson, R. M.; Dennis, T. J. S.; Hare, J. P.; Prassides, K. Europhys. Lett. 1992, 18, 219. 


\section{Figure Captions}

1. Schematic of TPA templating in sol-gel silica. Organic-inorganic hybrid silica formed by incorporation of TPA within a sol-gel silica matrix (left), and TPA-templated microporous silica formed after removal of TPA by calcination (right).

2. The intermolecular ${ }^{1} \mathrm{H}-{ }^{29} \mathrm{Si}$ cross-polarization spectra of the bulk and thin film xerogels recorded using $1 \mathrm{~ms}$ and $3 \mathrm{~ms}$ cross-polarization times. 

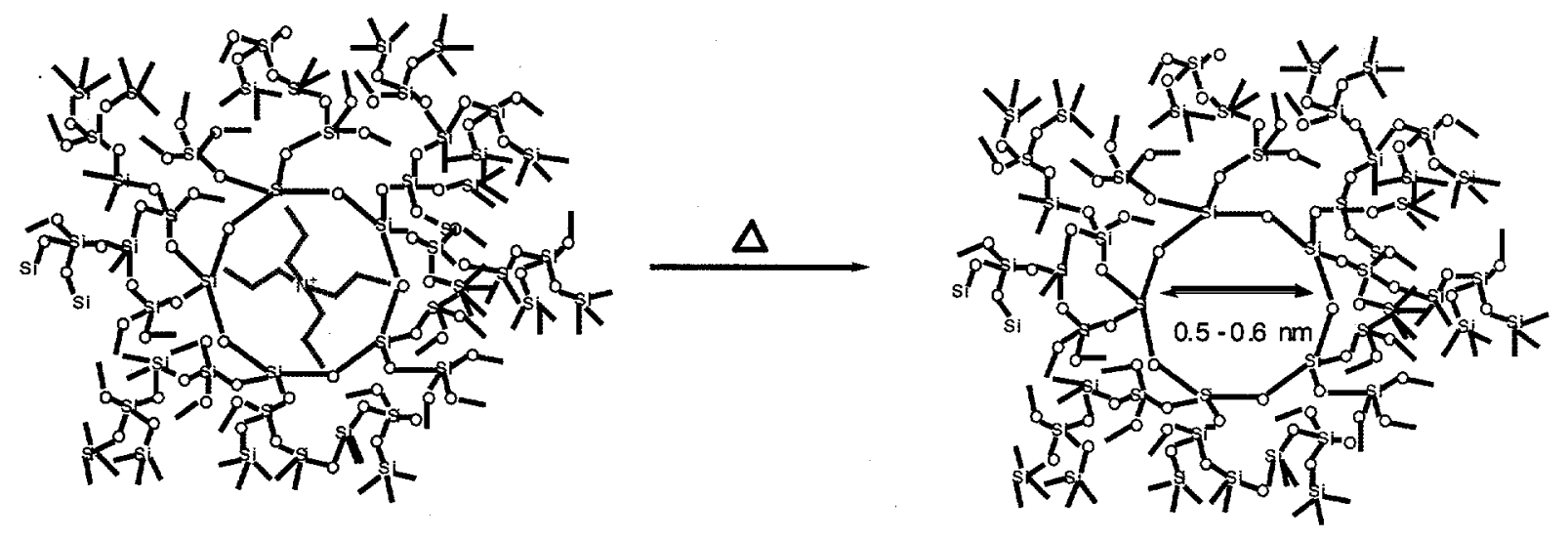

Figure 1 


\section{Bulk Xerogel}
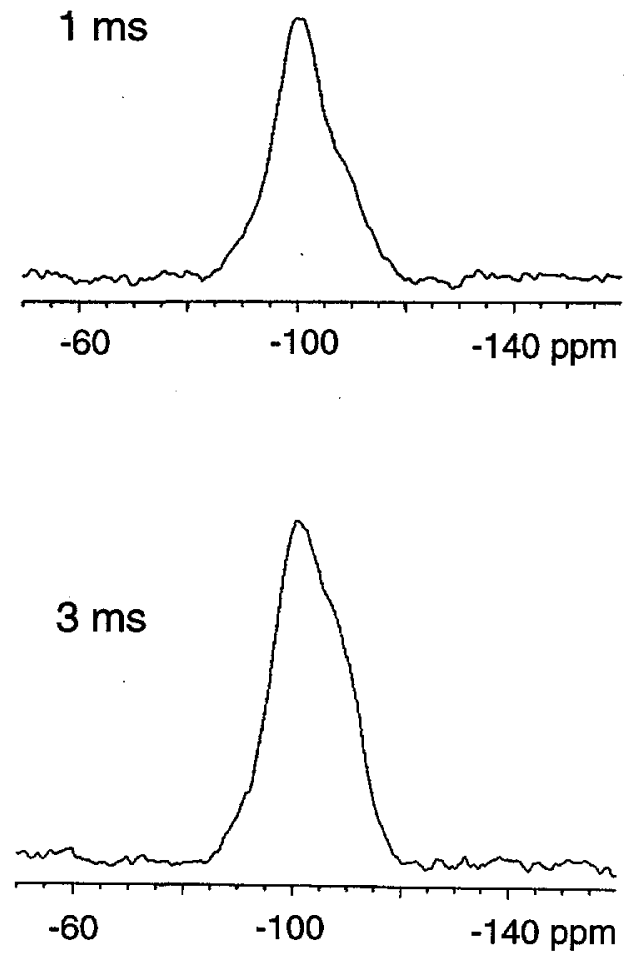

Thin Film Xerogel
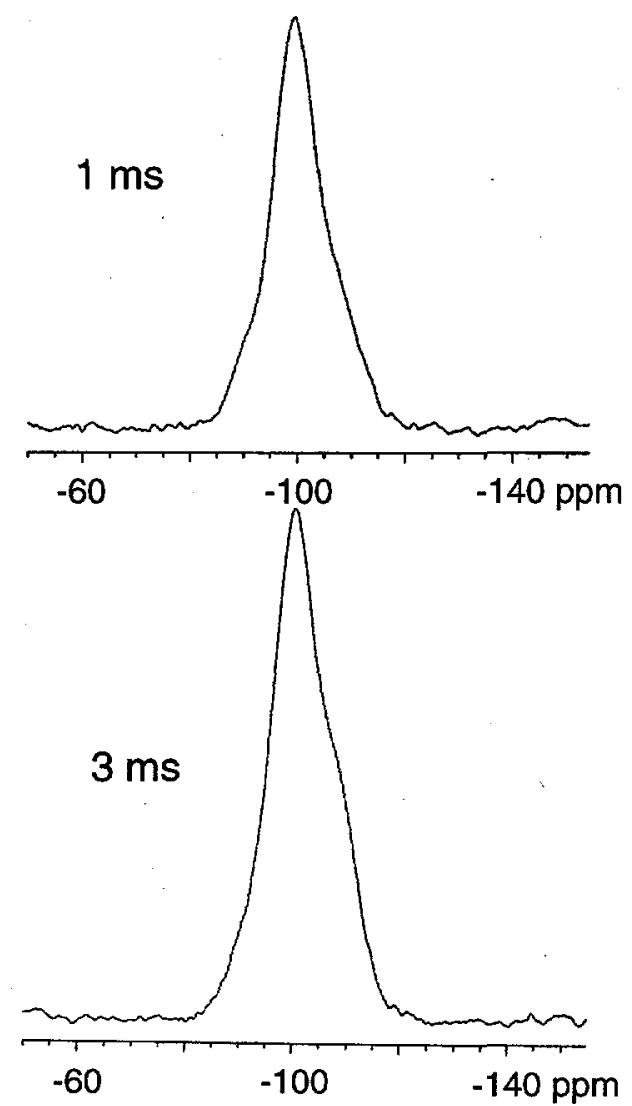

Figure 2 
Table I. The Relative Intensities of ${ }^{1} \mathrm{H}^{-29} \mathrm{Si}$ Intermolecular Cross-Polarization NMR Spectra of Bulk and Thin Film Xerogels.

\begin{tabular}{|c|c|c|c|}
\hline & Bulk Xerogels (1) & Thin Film Xerogels (2) & Ratio $(2) /(1)$ \\
\hline Contact Time & \multicolumn{3}{|c|}{${ }^{1} \mathrm{H}^{-29} \mathrm{Si}$ Intermolecular $\mathrm{CP}$, trial\# 1} \\
\hline $1 \mathrm{~ms}$ & 0.73 & 1.05 & 1.44 \\
\hline \multirow[t]{2}{*}{$3 \mathrm{~ms}$} & 1.00 & 1.35 & 1.35 \\
\hline & \multicolumn{3}{|c|}{${ }^{1} \mathrm{H}-{ }^{29} \mathrm{Si}$ Intermolecular $\mathrm{CP}$, trial\# 2} \\
\hline $1 \mathrm{~ms}$ & 0.80 & 1.01 & 1.26 \\
\hline \multirow[t]{3}{*}{$3 \mathrm{~ms}$} & 1.00 & 1.31 & 1.31 \\
\hline & \multirow{2}{*}{\multicolumn{2}{|c|}{$\begin{array}{l}\text { Average } \\
\text { Average, Normalized by TPA Concentration }\end{array}$}} & 1.34 \\
\hline & & & 1.35 \\
\hline
\end{tabular}

All intensities corrected for actual sample weights. 
Table II. The Relative Intensities of ${ }^{13} \mathrm{C}$ Direct and ${ }^{1} \mathrm{H}-{ }^{13} \mathrm{C}$ Intermolecular Cross-Polarization NMR Spectra of Bulk and Thin Film Xerogels.

\begin{tabular}{|c|c|c|c|}
\hline & Bulk Xerogels (1) & Thin Film Xerogels (2) & Ratio (2)/(1) \\
\hline $\begin{array}{c}\text { Contact Time } \\
10 \mathrm{~s}\end{array}$ & 0.94 & $\begin{array}{c}{ }^{13} \mathrm{C} \text { Direct Polarization } \\
0.98\end{array}$ & 1.04 \\
\hline \multirow[t]{2}{*}{$20 \mathrm{~s}$} & 1.00 & 0.94 & 0.94 \\
\hline & Average & & 0.99 \\
\hline & & ${ }^{1} \mathrm{H}-{ }^{13} \mathrm{C}$ Intramolecular $\mathrm{CP}$ & \\
\hline $0.3 \mathrm{~ms}$ & 1.19 & 1.46 & 1.23 \\
\hline \multirow[t]{3}{*}{$1.0 \mathrm{~ms}$} & 1.00 & 1.30 & 1.30 \\
\hline & Average & & 1.26 \\
\hline & Average, Normalize & by TPA Concentration & 1.27 \\
\hline
\end{tabular}

All intensities corrected for actual sample weights. 